\title{
MODELING OF CURRENT TRANSFER IN AlAs/GaAs HETEROSTRUCTURES WITH ACCOUNTING FOR INTERVALLEY SCATTERING
}

\author{
Nataly A. Vetrova, Yuri A. Ivanov, Evgeny V. Kuimov, Mstislav O. Makeev, Sergey A. \\ Meshkov, Kirill P. Pchelintsev, Vasily D. Shashurin
}

Bauman Moscow State Technical University, http://www.bmstu.ru

Moscow 105005, Russian Federation

vetrova@bmstu.ru,y-a-ivanov@mail.ru, ekjmo@mail.ru, mc.stiv@gmail.com,sb67241@mail.ru,pkp@ bmstu.ru, schashurin@bmstu.ru

Abstract. When modeling devices based on AlAs/GaAs heterostructures, an important factor to consider is intervalley electron scattering at heterointerfaces. This paper shows an approach to modeling such processes using the Schrödinger equations for the open reservoir-channel-reservoir system. The problem of modeling the intervalley scattering of electrons in the case when the profile of the aluminum fraction is continuous when there are no pronounced heterointerfaces is considered. For the numerical solution of the equations obtained, the method of finite differences is used without reducing the accuracy of the difference scheme to the first order when boundary conditions are considered. The methods of composing the elements of the matrices of system of linear equations to reduce the temporal complexity of the algorithm are considered. Based on the results of the calculation in the two-valley approximation, in comparison with singlevalley calculations, it was concluded that this model can be used to calculate the current-voltage characteristics of AlAs/GaAs heterostructures, taking into account the manufacturing technology and the patterns of degradation during operation, and also in evaluating the reliability parameters of nanoelectronics devices.

Keywords: heterostructures, nanoelectronic, math modeling, electrons intervalley scattering, twovalley approximation

UDC 004.052, 538.91

Bibliography - 9 references

Received 18.05.2018

RENSIT, 2018, 10(1):71-76

DOI: $10.17725 /$ rensit.2018.10.071

\section{Contents}

1. INTRODUCTION (71)

2. EXPERIMENTAL PART (72)

3. Results and discussion (74)

4. Conclusion (75)

REFERENCES (76)

\section{INTRODUCTION}

Nanoelectronic devices based on tunnel effects have extended (in comparison with traditional devices) functionality associated with the unique form of current-voltage characteristics (CVC), and are characterized by high perfomance. Such devices play an important role in the development of ultra-highspeed integrated microcircuits with an ultra-high degree of integration [1]. Resonance-tunnel diodes (RTD) and GaAs/AlAs-heterostructure transistors are used both in analog and digital integrated circuits as elements with CVC with a negative differential resistance section (for example, in ultrafast logic gates, whose operation is based on the transition from monostable to bistable state of monostablebistable transition logic elements - MOBILEs [2-3], in SRAM random access memory cells), and also as elements providing the possibility of optimizing the curvature of the initial section of the CVC by a criterion based on the performance characteristics of the device (for example, in frequency mixer). The high potential AlAs-barriers of the RTD heterostructures determine the increased operating temperature range: on the one hand, the tunnel current is reduced while the barrier thickness remains unchanged; on the other, the current associated with thermal excitation and above-barrier emission of electrons into the quantum well is reduced. The use of wide bandgap semiconductors as barrier material ensures the reliable functioning of such structures at room temperatures [4]. However, when modeling 
such instruments, it should be taken into account that AlxGa1-xAs at $\mathrm{x}>0.45$, including AlAs, are in direct bandgap semiconductor, unlike the direct bandgap GaAs and the AlxGa1-xAs at $x<0.45$. The lowest in AlAs is the X-valley, and in GaAs is the $\Gamma$-valley. The AlAs/GaAs-heterostructure layers are most often grown on a substrate with a crystallographic orientation [100], in the same direction, the electrons are accelerated by an external field. So, the intervalley scattering of electrons from the $\Gamma$-valleys of GaAs into X-valleys of AlAs on heterointerfaces is most likely at crystallographic direction [100]. The low probability of scattering in other directions and in other valleys is confirmed by calculations and causes the low efficiency of such multi-band models with increased computational complexity [5]. Intervalley $\Gamma \mathrm{X}$-scattering is modeled by writing a separate Schrödinger equation for the $\Gamma$-valley and the $\mathrm{X}$-valley, and each operator introduces a communication operator acting on the $\mathrm{X}$-valley wave function for the $\Gamma$-valley equation and vice versa [5-7]. If the assumption is made that the profile of the $\mathrm{Al}$ molar fraction at the heterointerface is a step function, then the communication operator at this heterointerface represents a delta function multiplied by the intervalley interaction constant $[5,6]$. In the case that the problem of accounting for diffusion changes in the profile of the molar fraction of $\mathrm{Al}$ is required, the assumption of its discontinuous change at the heterointerface is not generally equitable. That problem is appearing when assessing the reliability of nanoelectronics devices during operation under conditions of hard external influences. Therefore, the question arises of generalizing the definition of the communication operator to the case of a smoothly varying profile of the molar fraction of $\mathrm{Al}$ and in general the construction of a more general numerical model of current transfer with allowance for intervalley scattering.

\section{EXPERIMENTAL PART}

To conduct a numerical experiment, we formulate a mathematical model of current transfer within the framework of the wave function formalism. In the one-valley approximation, the motion of an electron in the active region is described by the stationary Schrödinger equation with a variable effective mass.
Bastard boundary conditions act on the boundary of the modeled region with electronic reservoirs.

$$
\left\{\begin{array}{l}
-\frac{\hbar^{2}}{3} \frac{d}{d z} \frac{1}{m^{*}} \frac{d}{d z} \psi+U \psi=E \psi, \\
\psi\left(z_{L}\right)=A_{L} e^{i k_{L} z_{L}}+B_{L} e^{-i k_{L} z_{L}}, \\
\psi\left(z_{R}\right)=A_{R} e^{i k_{R} z_{R}}+B_{R} e^{-i k_{R} z_{R}}, \\
\frac{1}{m_{L}^{*}} \frac{d}{d z} \psi\left(z_{L}\right)=\frac{i k_{L}}{m^{*}\left(z_{L}\right)} A_{L} e^{i k_{L} z_{L}}-\frac{i k_{L}}{m^{*}\left(z_{L}\right)} B_{L} e^{-i k_{L} z_{L}}, \\
\frac{1}{m_{R}^{*}} \frac{d}{d z} \psi\left(z_{R}\right)=\frac{i k_{R}}{m^{*}\left(z_{R}\right)} A_{R} e^{i k_{R} z_{R}}-\frac{i k_{R}}{m^{*}\left(z_{R}\right)} B_{R} e^{-i k_{R} z_{R}},
\end{array}\right.
$$

where $\hbar$ - reduced Planck constant, $z$ - coordinate, $m^{*}$ $=m^{*}(z)$-effective mass, $\psi$ - electrons wave function, $U=U(z)$ - electrons potential energy, $E$ - electrons total energy, $\tau_{L(R}-$ left (right) bound of modeling region, $A_{L(\mathbb{R})}$ and $B_{L(\mathbb{R}}$ - electrons waves amplitudes in left (right) reservoir, $k_{L(R)}=\frac{\sqrt{2 m_{L(R)}^{*}\left(E-U_{L(R)}\right)}}{\hbar}-$ electrons wave number in left (right) reservoir, $m_{L(R)}^{*}$ - electrons effective mass in left (right) reservoir.

The potential energy of the electron $U(z)$ is defined as the sum of the profile of the bottom of the conduction band and the potential energy of the external field due to the voltage applied to the structure.

$U(z)=E_{c}-q_{e} V$,

where $E_{c}-$ the profile of the bottom of the conduction band, $V$ - potential of the external field, $q_{\mathrm{e}}$ - elementary charge.

In the case of AlGaAs-heterostructures, the bottom profiles of the conduction band and the effective mass of an electron are determined by the molar fraction of $\mathrm{Al}$.

The system (1) is rather cumbersome, however, one can write an equation equivalent to this system [8-9]

$$
\left[\frac{d}{d z} \frac{\hbar^{2}}{2 m^{*}} \frac{d}{d z}+(E-U)+\Sigma_{L}+\Sigma_{R}\right] \psi=S_{L}+S_{R},
$$

where

$$
\begin{aligned}
& \Sigma_{L(R)}=i k_{L(R)} \frac{\hbar^{2}}{2 m_{L(R)}^{*}} \delta\left(z-z_{R}\right), \\
& S_{L}=-2 i k_{L} \frac{\hbar^{2}}{2 m_{L}^{*}} \delta\left(z-z_{L}\right) A_{L} \\
& S_{R}=-2 i k_{R} \frac{\hbar^{2}}{2 m_{R}^{*}} \delta\left(z-z_{R}\right) B_{R}
\end{aligned}
$$


Equation (3) is not only more compact than system (1), but it is also more convenient from the point of view of physical interpretation. Thus, the appearance of the operators $\Sigma_{\mathrm{L}}$ and $\Sigma_{\mathrm{R}}$ is explained by the interaction of the channel with the reservoirs, in other words, we can assume that these operators describe the outflow of electrons from the channel. At the same time, the quantities $S_{\mathrm{L}}$ and $S_{\mathrm{R}}$ are interpreted as terms describing the injection of electrons from the reservoirs into the channel.

To describe the motion of electrons with allowance for the intervalley scattering, it is necessary to write down two Schrödinger equations for each valley.Both these equations contain terms describing the relation between valleys

$$
\left\{\begin{array}{l}
{\left[\frac{d}{d z} \frac{\hbar^{2}}{2 m^{\Gamma}} \frac{d}{d z}+\left(E-U^{\Gamma}\right)+\Sigma_{L}^{\Gamma}+\Sigma_{R}^{\Gamma}\right] \psi^{\Gamma}+A \psi^{X}=S_{L}^{\Gamma}+S_{R}^{\Gamma},} \\
{\left[\frac{d}{d z} \frac{\hbar^{2}}{2 m^{X}} \frac{d}{d z}+\left(E-U^{X}\right)+\Sigma_{L}^{X}+\Sigma_{R}^{X}\right] \psi^{X}+A \psi^{\Gamma}=S_{L}^{X}+S_{R}^{X} .}
\end{array}\right.
$$

The system (5) describes the motion of the electrons already taking into account the $\Gamma-\mathrm{X}$ scattering. The superscript $\Gamma(\mathrm{X})$ denotes the membership of a physical quantity to the $\Gamma(\mathrm{X})$-value. Communication operator A in case a GaAs/AlAsheterostructures is defined in the following way

$$
A=\alpha \sum_{k=1}^{M+1} \delta\left(z-z_{k}\right)
$$

where $\alpha$ - intervalley interaction constant, $\gamma_{\mathrm{k}}-$ coordinate $k$ 'th-heteroboundary, $M$ - number of layers in a heterostructure, $\delta(z)$ - delta function.

Usually, in simulation, it is assumed that all electrons fall into the active region from the $\Gamma$-valley. Therefore, terms of sources $S_{L}^{X}=S_{R}^{X}=0$. .

Communication operator definition (6) is rightly only in case when profile of the heterointerface is clearly expressed, i.e., when the profile of the fraction $\mathrm{Al}$ at the heterointerfaces is a step function. In the event that the profile of the fraction $\mathrm{Al}$ is a continuous function, a generalization of the definition of the communication operator is required, for example, in accordance with formula (7)

$$
A=\alpha\left|\frac{d}{d z} x\right|,
$$

where $x=x(z)-A l$ molar fraction.

The system of equations (5) in the general case is solved only numerically. For a numerical solution of this system, we use the method of finite differences on a regular mesh, with the help of which we obtain a system of linear algebraic equations

$$
\left\{\begin{array}{l}
t_{i-1}^{\Gamma} \psi_{i-1}^{\Gamma}+\left(E-U_{i}^{\Gamma}+\Sigma_{i}^{\Gamma}-t_{i-1}^{\Gamma}-t_{i}^{\Gamma}\right) \psi_{i}^{\Gamma}+t_{i}^{\Gamma} \psi_{i}^{\Gamma}+A_{i} \psi_{i}^{X}=S_{i}^{\Gamma}, \\
t_{i-1}^{X} \psi_{i-1}^{X}+\left(E-U_{i}^{X}+\Sigma_{i}^{X}-t_{i-1}^{X}-t_{i}^{X}\right) \psi_{i}^{X}+t_{i}^{X} \psi_{i}^{X}+A_{i} \psi_{i}^{\Gamma}=S_{i}^{X} .
\end{array}\right.
$$

The coefficients in front of the node values of the wave function $\psi_{i}^{\Gamma(X)}$ are defined as follows

$$
\begin{aligned}
& t_{i}^{\Gamma(X)}=\frac{\hbar^{2}}{2 m_{i}^{\Gamma(X)} h^{2}}, A_{i}=\frac{1}{2 h} \int_{z_{i-1}}^{z_{i+1}} A d z, \\
& m_{i}^{\Gamma(X)}=\frac{1}{h} \int_{z_{i}}^{z_{i+1}} m^{\Gamma(X)} d z, \quad U_{i}^{\Gamma(X)}=\frac{1}{2 h} \int_{z_{i-1}}^{z_{i+1}} U^{\Gamma(X)} d z, \\
& \Sigma_{i}^{\Gamma(X)}=\frac{1}{2 h} \int_{z_{i-1}}^{z_{i+1}}\left(\Sigma_{L}^{\Gamma(X)}+\Sigma_{R}^{\Gamma(X)}\right) d z, \\
& \mathrm{~S}_{i}^{\Gamma(X)}=\frac{1}{2 h} \int_{z_{i-1}}^{z_{i+1}}\left(S_{L}^{\Gamma(X)}+S_{R}^{\Gamma(X)}\right) d z,
\end{aligned}
$$

where $h$ - mesh spacing.

By definition, and given that $S_{L}^{X}=S_{R}^{X}=0$

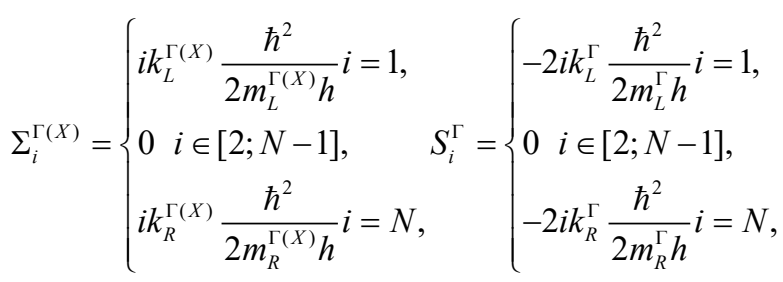

$S_{i}^{X}=0$,

where $k_{L(R)}^{\Gamma(X)}$ - electron wavenumber in $\Gamma(\mathrm{X})$-valley in left (right) reservoir is defined analogously to the one-valley case.

We note that the system of equations (8) can be used as in the case of stepwise and in the case of a continuous change in the profile of $\mathrm{Al}$ at heterojunctions.

The system (8) can be written in matrix form. Depending on the layout of the equations, you can do this in two ways. We write the system (8) for a mesh of three nodes

$\left(\begin{array}{llllll}d_{1}^{\Gamma} & \mathrm{t}_{1}^{\Gamma} & 0 & A_{1} & 0 & 0 \\ t_{1}^{\Gamma} & d_{2}^{\Gamma} & t_{2}^{\Gamma} & 0 & A_{2} & 0 \\ 0 & t_{2}^{\Gamma} & d_{3}^{\Gamma} & 0 & 0 & A_{3} \\ A_{1} & 0 & 0 & d_{1}^{X} & t_{1}^{X} & 0 \\ 0 & A_{2} & 0 & t_{1}^{X} & d_{2}^{X} & t_{2}^{X} \\ 0 & 0 & A_{3} & 0 & t_{2}^{X} & d_{3}^{X}\end{array}\right)\left(\begin{array}{l}\psi_{1}^{\Gamma} \\ \psi_{2}^{\Gamma} \\ \psi_{3}^{\Gamma} \\ \psi_{1}^{X} \\ \psi_{2}^{X} \\ \psi_{3}^{X}\end{array}\right)=\left(\begin{array}{c}S_{1}^{\Gamma} \\ S_{2}^{\Gamma} \\ S_{3}^{\Gamma} \\ S_{1}^{X} \\ S_{2}^{X} \\ S_{3}^{X}\end{array}\right)$,




$$
\left(\begin{array}{cccccc}
d_{1}^{\Gamma} & A_{1} & t_{1}^{\Gamma} & 0 & 0 & 0 \\
A_{1} & d_{1}^{X} & 0 & t_{1}^{X} & 0 & 0 \\
t_{1}^{\Gamma} & 0 & d_{2}^{\Gamma} & A_{2} & t_{2}^{\Gamma} & 0 \\
0 & t_{1}^{X} & A_{2} & d_{2}^{X} & 0 & t_{2}^{X} \\
0 & 0 & t_{2}^{\Gamma} & 0 & d_{3}^{\Gamma} & A_{3} \\
0 & 0 & 0 & t_{2}^{X} & A_{3} & d_{3}^{X}
\end{array}\right)\left(\begin{array}{l}
\psi_{1}^{\Gamma} \\
\psi_{2}^{X} \\
\psi_{2}^{\Gamma} \\
\psi_{2}^{X} \\
\psi_{3}^{\Gamma} \\
\psi_{3}^{X}
\end{array}\right)=\left(\begin{array}{c}
S_{1}^{\Gamma} \\
S_{2}^{X} \\
S_{2}^{\Gamma} \\
S_{2}^{X} \\
S_{3}^{\Gamma} \\
S_{3}^{X}
\end{array}\right),
$$

where $d_{i}^{\Gamma(X)}=E-U_{i}^{\Gamma}+\Sigma_{i}^{\Gamma}-t_{i-1}^{\Gamma}-t_{i}^{\Gamma}$.

For larger dimensions, the layout method (12) is more efficient when using the Thomas algorithm.

\section{RESULTS AND DISCUSSION}

This mathematical model was tested on a model structure, the parameters of which are indicated in Table 1. The simulation was conductt for different values of the intervalley simulation constant for the purpose of investigating the effect of this quantity on the calculated CVC.

In Fig. 1 and Fig. 2 the results of calculation of wave functions in the $\Gamma$-valley and $\mathrm{X}$-valley in the structure channel from Tab. 1. For the convenience of perception, the natural logarithm of the squares of the moduli of the wave functions is mapped. The black transparent surface shows the profile of the bottom of the conduction band in the corresponding valley.

As can be seen from the figures, when calculating in the two-valley approximation, new metastable levels appear in the $\Gamma$-valley, corresponding to metastable levels in the $\mathrm{X}$-valley. This explains the appearance of new peaks in the tunnel transparency coefficient, the graphs of which are presented in Fig. 3.

Table 1.

\section{Modelstructure parameters}

\begin{tabular}{|c|c|c|c|}
\hline $\begin{array}{c}\text { Thickness, } \\
\mathrm{nm}\end{array}$ & Material & $\begin{array}{c}\text { Density of } \\
\mathrm{Si}, 10^{22} \mathrm{~m}^{-3}\end{array}$ & Comment \\
\hline 50 & $\mathrm{GaAs}$ & 400 & Contactlayer \\
\hline 5 & $\mathrm{GaAs}$ & 0 & Spacer \\
\hline 2 & $\mathrm{AlAs}$ & 0 & Barrier \\
\hline 3 & $\mathrm{GaAs}$ & 0 & Well \\
\hline 2 & $\mathrm{AlAs}$ & 0 & Barrier \\
\hline 5 & $\mathrm{GaAs}$ & 0 & Spacer \\
\hline 50 & $\mathrm{GaAs}$ & 400 & Contactlayer \\
\hline
\end{tabular}

Fig. 3. The coefficient of tunnel transparency at $a=0.01$
$\mathrm{eV} \cdot \mathrm{nm}$ (upper) $a=0.05 \mathrm{eV} \cdot \mathrm{nm}$ (average) and $a=0.10$
$\mathrm{eV} \cdot \mathrm{nm}$ (lower). The gray line shows the tunneling transparency

Fig. 3. The coefficient of tunnel transparency at $a=0.01$
$\mathrm{eV} \cdot \mathrm{nm}$ (upper) $a=0.05 \mathrm{eV} \cdot \mathrm{nm}$ (average) and $a=0.10$
$\mathrm{eV} \cdot \mathrm{nm}$ (lower). The gray line shows the tunneling transparency

Fig. 3. The coefficient of tunnel transparency at $a=0.01$
$\mathrm{eV} \cdot \mathrm{nm}$ (upper) $a=0.05 \mathrm{eV} \cdot \mathrm{nm}$ (average) and $a=0.10$
$\mathrm{eV} \cdot \mathrm{nm}$ (lower). The gray line shows the tunneling transparency coefficient in the one-valley approximation.

Fig. 1. Logarithm of the square of the modulus of the electron wave function in the $\Gamma$-valley or $a=0 \mathrm{eV} \cdot \mathrm{nm}$.
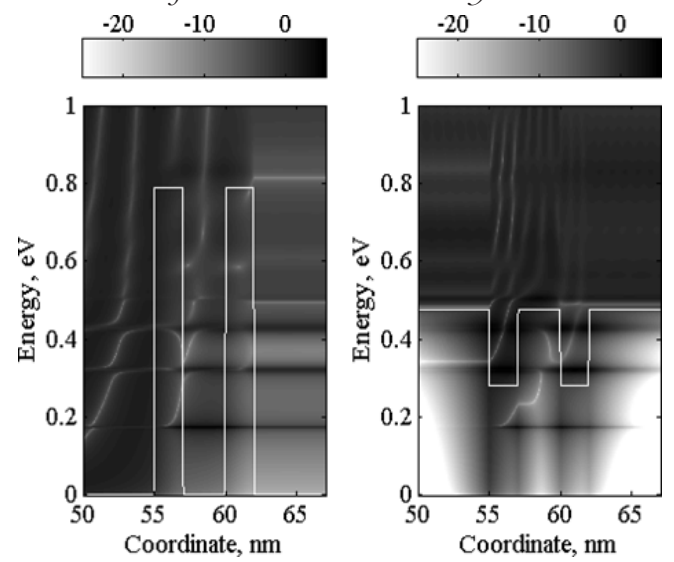

Fig. 2. The logarithm of the square of the modulus of the electron wave function in the $\Gamma$-valley (on the left) and in the $X$-valley (on the right) for $a=0.05 \mathrm{eV} \cdot \mathrm{nm}$.

With an increase in the intervalley interaction constant, the new maxima of the tunneling transparency coefficient are broadened and the "one-valley" maxima are displaced. From this it can be concluded that when the intervalley model is increased, the peak of the current density on the
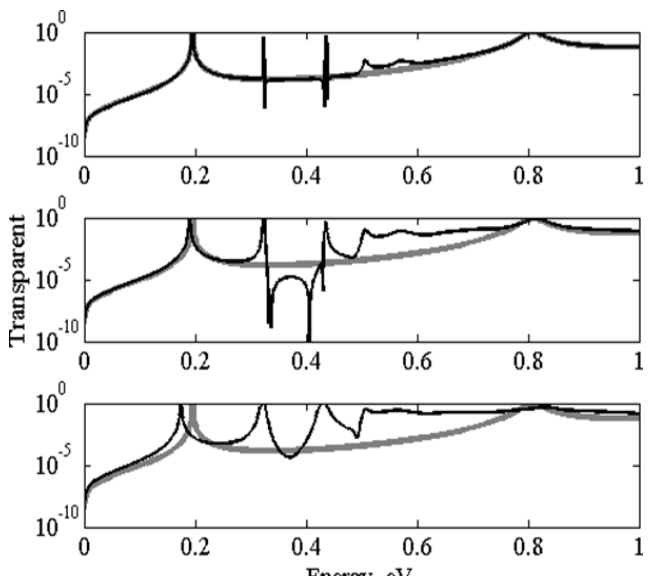$$
\text { coefrient in the one-valley appraximation. }
$$ 


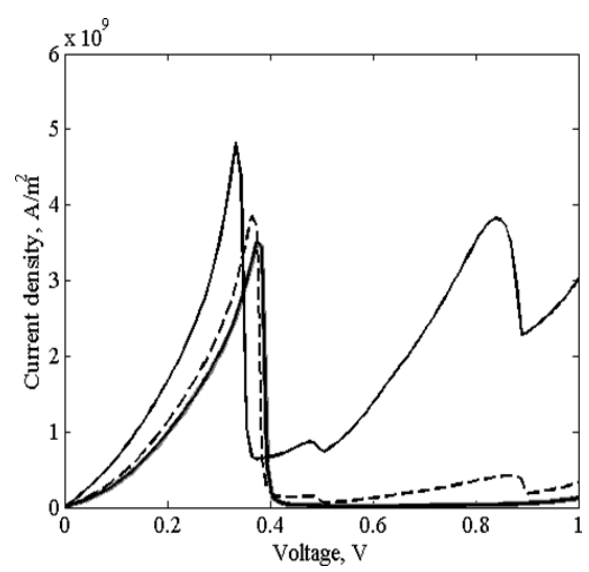

Fig. 4. CVC of the model structure at $a=0.01 \mathrm{eV} \cdot \mathrm{nm}$ (solid) $a=0.05 \mathrm{eV} \cdot \mathrm{nm}$ (dotted) and $a=0.10 \mathrm{eV} \cdot \mathrm{nm}$ (dot-dash). The gray line shows the CVC in a one-valley approximation.

calculated CVC will shift towards lower voltages and its value will increase. Also, new maximums will appear on the CVC, which we see on the graphs in Fig. 4.

The presented method of calculating the tunneling transparency coefficient in the two-valley approximation was used in the implementation of the algorithm for modeling heterostructures in the MATLAB. To entering the parameters of the heterostructure and to represent the output parameters $(\mathrm{CVC}$, tunneling transparency coefficient, etc.), a graphical interface was developed. In addition to the intervalley scattering, the implemented model takes into account the electron-electron interaction by the self-consistent field method, inelastic electron scattering by the optical potential method and parasitic resistance of the contact regions. In Fig. $\mathbf{5}$ a window of the interface with the results of the calculation of the CVC is shown taking into account the self-consistent field and intervalley dispersion. In the plot, the dark line corresponds to the CVC,

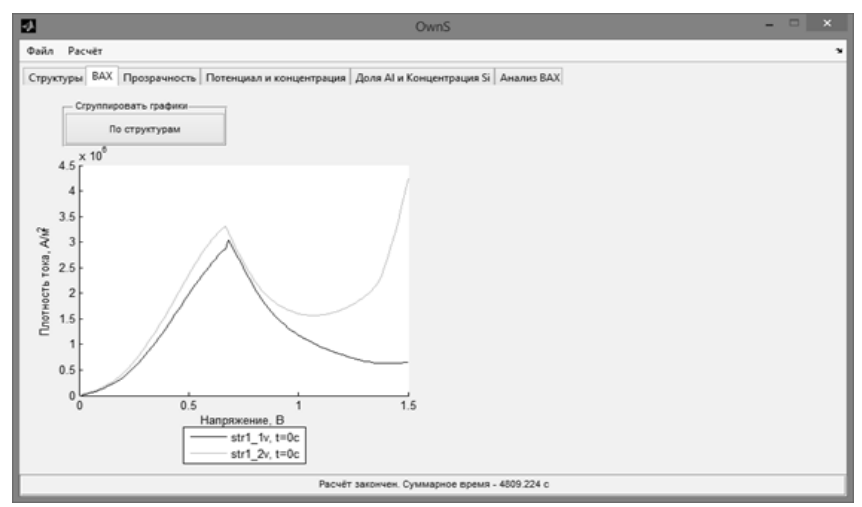

Fig. 5. CVC of the model structure. calculated in the one-valley approximation, and the light line in the two-valley approximation.

On the plot of the calculated CVC, the same trends are seen as in Fig. 4, namely, an increase in the peak current density and a decrease in the peak voltage. Also on this CVC, due to the consideration of intervalley scattering, a pronounced second region of positive differential conductivity appears, which is also present on the experimentally measured CVC of resonance-tunnel structures.

\section{CONCLUSION}

The Schrodinger equation with Bastard boundary conditions was reduced to the Schrodinger equation for an open system, which improves the physical interpretation of processes in the structure channel.A system of two such equations, supplemented by a communication operator, makes it possible to describe the motion of an electron in a channel with allowance for intervalley scattering.A system of two such equations supplemented by a communication operator allows for the modeling of current transfer in heterostructures, taking into account diffusion processes, the definition of a communication operator for the description of AlGaAsheterostructures with the characteristic behavior of the $\mathrm{Al}$ profile was expanded, taking into account the influence of the technological process of their manufacture and the degradation patterns during the operation of devices on their basis.A numerical model was obtained, obtained within the framework of the finite difference method without reducing the accuracy of the difference scheme to the first order, taking into account the boundary conditions. The time complexity of the implemented algorithm is reduced due to the choice of the method for forming the coefficient matrix of a system of linear equations. The calculation of the $\mathrm{CVC}$ of the model structure was carried out for different values of the intervalley interaction constant. The developed model was integrated into AlGaAsheterostructure modeling algorithm.Based on the results of the calculation in the two-valley approximation in comparison with the one-valley calculations, it was concluded that it is expedient to use this model in the calculationCVCAlAs/GaAs-heterostructureswith taking into account the patterns of degradation in the process of operation under conditions of exposure to ionizing radiations of various nature and elevated 
temperature in evaluating the reliability parameters of nanoelectronics devices.

The work was supported by the Ministry of Education and Science of the Russian Federationproject № 16.1663.2017/4.6.

\section{REFERENCES}

1. Ivanov YuA, Meshkov SA, Shashurin VD, FedorkovaNV,FedorenkoIA.Subgarmonicheskiy smesitel suluchshennymi intermodulyatsionnymi kharakteristikami na baze rezonansnotunnelnogo dioda [Subharmonic mixer with improved intermodulation characteristics based on a resonance-tunnel diode]. Radiotekhnika $i$ elektronika, 2010, 55(8):982-988 (in Russ.).

2. Kim H, Seo K. Monostable-to-Bistable Transition Logic Element-Based Literal Gates Using Resonant Tunneling Diodes. Japanese Journal of Applied Physics, 2008, 47(5):3340-3345.

3. Arun Goud Akkala. NEGF simulation of electron transport in resonant tunneling and resonant interband tunneling diodes. Dissertation degree M.S.E.C.E, 2011, 80 p.

4. Borisenko VE, Ossicini S. What is What in the Nanoworld. Third, Revised and Enlarged Edition. Wiley-VCH, Weinheim, 2012, 601 p.

5. Abramov II, Goncharenko IA, Kolomeytseva NV. Kombinirovannaya dvukhzonnaya model rezonansno-tunnelnogo dioda [Combined twoband model of a resonance-tunnel diode]. Fizikea i tekhnike a poluprovodnikov, 2007, 41(11):1395-1400 (in Russ.).

6. Sun JP, Mains RK, Yang K, Haddad GI. A SelfConsistent Model of $\Gamma$-X Mixing in GaAs/ AlAs/GaAs Quantum Well Using Quantum Transmitting Boundary Method. J. Appl. Phys., 1993, 74(8):5053-5060.

7. Fedyay A., Moskaliuk V. Hyerarchial Approach to Resonant-Tunneling Diode Modeling. ELNANO' 2012, April 10-12, 2012, Kyiv, Ukraine, p.58-59.

8. Datta S. Electronic Transport in Mesoscopic Systems. Cambridge University Press, Cambridge, 1995.

9. Datta S. Quantum Transport. Atom to Transistor. Cambridge University Press, Cambridge, 2005, $404 \mathrm{p}$. 